\title{
Incidência de gravidez na adolescência no período pandêmico nas Unidades Básicas de Saúde em um município da região sul do Estado do Tocantins
}

\author{
Incidence of teenage pregnancy in the pandemic period in basic health units in a city from \\ the south region of Tocantins state
}

Incidencia del embarazo en la adolescencia en el período de pandemia en unidades básicas de salud de uma ciudad de la región sur del estado de Tocantins

Dayanne Ribeiro de Sá1*, João Marcelo Alves de Souza ${ }^{1}$, Maryane Ribeiro Sales ${ }^{1}$, Sandra Nara Marroni ${ }^{1}$.

\section{RESUMO}

Objetivo: Conhecer a incidência de gravidez na adolescência no período pandêmico em Unidades Básicas de Saúde, bem como, caracterizá-las biossocialmente em uma cidade ao sul do Estado do Tocantins. Métodos: Trata-se de uma pesquisa de campo, exploratória, descritiva com abordagem quantitativa. Foi realizada exclusivamente nos prontuários das gestantes de todas as Unidades Básicas de Saúde envolvidas, sendo excluídas gestantes acompanhadas por qualquer rede privada. O estudo foi aprovado pelo Comitê de Ética e Pesquisa sob o parecer número 4.444.659. Resultados: A amostra revelou uma incidência de 17,34\% de casos de gravidez na adolescência no período proposto, a maioria das adolescentes com idade entre 16 e 18 anos $(59,85 \%)$, pardas $(30,30 \%)$, solteiras $(41,67 \%)$, escolaridade predominante Ensino Fundamental incompleto (18,93\%), não planejaram sua gravidez $(45,45 \%)$ e não tiveram abortos anteriores $(70,45 \%)$. Conclusão: Concluiu-se que a incidência não diminuiu no período pandêmico e as demais variáveis também se mantiveram equivalentes a outras pesquisas.

Palavras-chave: Gravidez na adolescência, Pandemia, Saúde pública.

\begin{abstract}
Objective: Know the incidence of teenage pregnancy in the pandemic period in Basic Health Units, as well as characterize them biosocially in a city in the south of Tocantins. Methods: This is an exploratory, descriptive field research with a quantitative approach. It was performed exclusively in the medical records of pregnant women from all the Basic Health Units involved, and pregnant women accompanied by any private network were excluded. The study was approved by the Ethics and Research Committee under opinion number 4.444.659. Results: The sample revealed an incidence of $17,34 \%$ of teenage pregnancy cases in the proposed period, most adolescents aged between 16 and 18 years old $(59.85 \%)$, brown (30.30\%), single $(41,67 \%)$, predominant education, incomplete Elementary School $(18.93 \%)$, did not plan their pregnancy $(45.45 \%)$ and had no previous abortions $(70.45 \%)$. Conclusion: It was concluded that the incidence did not decrease in the pandemic period and the other variables also remained equivalent to other studies.
\end{abstract}

Keywords: Teenage pregnancy, Pandemic, Public health.

\section{RESUMEN}

Objetivo: Conocer la incidencia del embarazo adolescente en el período pandémico en Unidades Básicas de Salud, así como caracterizarlas biosocialmente en una ciudad del sur de Tocantins. Métodos: Se trata de una investigación de campo exploratoria, descriptiva con un enfoque cuantitativo. Se realizó exclusivamente en las historias clínicas de las gestantes de todas las Unidades Básicas de Salud involucradas y se excluyó a las

1 Universidade de Gurupi (UNIRG), Gurupi - TO. *E-mail: sandramarroni@gmail.com

PUBLICADO EM: 2/2022 
gestantes acompañadas de alguna red privada. El estudio fue aprobado por el Comité de Ética e Investigación con el número de dictamen 4.444.659. Resultados: La muestra reveló una incidencia de $17,34 \%$ de casos de embarazo adolescente en el período propuesto, la mayoría de adolescentes entre 16 y 18 años $(59,85 \%)$, morenas $(30,30 \%)$, solteras $(41,67 \%)$, educación predominante, primaria incompleta Escuela $(18,93 \%)$, no planificó su embarazo $(45,45 \%)$ y no tuvo abortos previos $(70,45 \%)$. Conclusión: Se concluyó que la incidencia no disminuyó en el período pandémico y las otras variables también se mantuvieron equivalentes a otros estudios.

Palabras clave: Embarazo adolescente, Pandemia, Salud pública.

\section{INTRODUÇÃO}

O termo "adolescência" advém da palavra adolescere, que possui origem latina e define-se como "crescimento" ou "processo de crescimento até a maturidade" (GROSSKLANS VK, 2019). É um período de crescimento e transições, que se caracteriza tanto por mudanças biológicas, sociais e psíquicas. Conforme $o$ Estatuto da Criança e do Adolescente (ECA) - Lei n $8069 / 1990$, adolescente é todo indivíduo com idade entre 12 e 18 anos. Entretanto, para a Organização Mundial de Saúde (OMS) esse período envolve indivíduos com idade entre 10 e 19 anos (MINISTÉRIO DA SAÚDE, 2007).

Segundo Silva PM e Milani DRC (2019), conjuntamente às transições do corpo, acontecem de acordo com uma ordem psicoemocional, que são vivenciadas de formas diversas, única a cada família ou sociedade. É importante salientar que além das modificações biopsicossociais, questões ligadas à maturação sexual, bem como, à busca da identidade adulta e ao desenvolvimento de autonomia perante os pais tornam-se fatores desencadeantes de uma redescoberta de algo intrínseco ao ser humano: a sexualidade (CUNHA ACS, 2020).

Ainda segundo Cunha ACS (2020) a interferência da cultura patriarcal, das escolas, da religião, bem como das questões políticas e econômicas podem intervir de maneira significativa na construção da sexualidade dos adolescentes. Visando tais interferências, o mencionado grupo acaba se tornando "de risco", tendo como consequência, na maioria das vezes, a prática de relações sexuais desprotegidas, no crescimento da vulnerabilidade às Infecções Sexualmente Transmissíveis (IST's), além da Gravidez na Adolescência (GA).

Para Costa MIF (2019) a cor/etnia, o nível de escolaridade, a estruturação da família, os aspectos socioeconômicos, as questões de gênero e os grupos sociais podem ter influência de maneira significativa com o precoce início da vida sexual de adolescentes. É de fundamental importância salientar as diferentes consequências na vida da adolescente grávida, tais como, a evasão escolar, a complexidade para ter acesso a um emprego, a possibilidade de não estar mais com o companheiro um ano após o parto. Destaca-se, além disso, as implicações psicossociais, como a aversão por parte da família, a insegurança, medo e vergonha da jovem, perda de sua liberdade e maiores riscos de depressão e suicídio (CUNHA ACS, 2020).

Nesse período de intenso desenvolvimento, vivenciarem a maternidade pode causar a interrupção de aspectos importantes em suas vidas. Essas mudanças na vida das jovens mães, ligadas à pouca idade para assumir essas responsabilidades relacionadas à maternidade, podem trazer uma percepção difícil e pouco satisfatória desta nova experiência, muitas vezes, obrigando a permanência no lar e a dependência familiar, podendo ser compreendido como uma prisão, ou algo negativo (ZACHI M, et al., 2016).

Cunha ACS (2020) diz, ainda, que são graves as consequências que podem afetar o filho da genitora adolescente, reforçando a situação de risco na qual mãe e filho podem se encontrar: parto prematuro, maior taxa de cesariana, aumento da morbimortalidade infantil, abandono e violência. Em países de baixa e média renda, calcula-se que, aproximadamente, 21 milhões de adolescentes de 15 a 19 anos engravidam e 16 milhões têm partos anualmente (SEHNEM DG, et al., 2019).

Contemporaneamente, a GA é considerada um problema social e, portanto, é alvo de estratégias biopolíticas no intuito de enfrentá-las. Observa-se que a gravidez precoce prejudica o desenvolvimento biológico, psicológico e social da adolescente, já que esta terá de assumir responsabilidades prematuramente (SILVA PM e MILANI DRC, 2019). 
Silva PM e Milani DRC (2019) afirmam que para assistir adolescentes com uma gestação não planejada ou não desejada é imprescindível a atenção dos profissionais em quatro situações: fundamentar-se em indícios científicos; realizar a sensibilização da comunidade (incluindo os adolescentes); ofertar informações adequadas e incentivar o diálogo com os envolvidos.

Recentemente, a doença causada pelo Severe Acute Respiratory Syndrome Coronavirus 2 (SARS-CoV2), a Coronavirus Disease 2019 (Covid-19), que emergiu no final de 2019 na cidade de Wuhan-China, tornouse a sexta emergência de saúde pública que requer atenção, tornando-se pandêmica. As medidas sanitárias de contenção para assegurar a proteção da população, retardar a propensão crescente de contaminação e evitar a crise nos serviços de saúde, se caracterizam como elementos que mudam o dia-a-dia das pessoas (OLIVEIRA WA, 2020). A OMS preconizou o isolamento social como método mais eficaz para inibir contaminação e mortes, uma vez que não há tratamento comprovadamente eficaz até o momento (FLORÊNCIO JÚNIOR PG, et al., 2020).

Embora a COVID-19 atinja pessoas de todas as idades, os níveis socioeconômicos, o sexo, a etnia e a vulnerabilidade a esta patologia estão intimamente relacionadas aos determinantes sociais do processo saúde/doença. Essa vulnerabilidade aumenta com as condições de vida, falta de dinheiro e o não acesso as políticas públicas essenciais, como saúde, educação e assistência social. Dessa forma, muitos adolescentes encontram-se em condição de alta vulnerabilidade, principalmente, nas periferias das cidades grandes. No momento em que se enfrenta uma doença pandêmica, precisa-se levar em consideração o entendimento, os significados concedidos à doença, a saúde, o risco, o acesso à prevenção, os cuidados, o tratamento e os aspectos presentes na abordagem dos adolescentes (OLIVEIRA WA, 2020).

Embora não se tenha indícios científicos que corroborem para o risco maior às gestantes e recém-nascidos quanto a COVID-19, diversas orientações procedem devido a semelhança com infecções ocasionadas por outros vírus, tais como, o SARS-CoV, o MIddle East Respiratory Syndrome (MERS-CoV), o H1N1 e todas as evidencias existentes até hoje estão sujeitas a alterações mediante geração de novos conhecimentos (WAGNER A, 2020). Assim sendo, o Ministério da Saúde (MS), preventivamente coloca as gestantes e puérperas, nas duas primeiras semanas após o parto, em grupo de risco para agravamento do quadro se forem contaminadas pelo vírus da COVID-19 (BRASIL, 2020).

Deste modo, no cenário da Atenção Primária em Saúde (APS), conhecer a vivência do adolescente sobre a sexualidade a partir dele mesmo, causa, para os profissionais, desafios para entender tais perspectivas e, a partir daí, utilizar as políticas e programas de educação sexual e reprodutiva em conjunto com as escolas e famílias desses indivíduos, ampliando os conhecimentos sobre assuntos decorrentes das experiências individuais e amplificando a rede de comunicação (SEHNEM DG, et al., 2019).

Logo este estudo buscou trazer dados sobre a incidência de grávidas adolescentes que fazem acompanhamento do pré-natal nas Unidades Básicas de Saúde (UBS) em um município da região sul do estado do Tocantins, no período de março de 2020 a fevereiro de 2021.

\section{MÉTODOS}

Trata-se de um estudo exploratório, descritivo de natureza quantitativa que foi realizado por meio de coleta de dados na rede pública de saúde, exclusivamente em prontuários das UBSs da zona urbana de um município da Região Sul do estado do Tocantins. Teve como a finalidade, conhecer a incidência de adolescentes gestantes entre o período de março de 2020 a fevereiro de 2021, período este que se entendia como início e possível final da pandemia da COVID-19.

Respeitando a ética e o sigilo do estudo, não contatou nenhum indivíduo. Dessa maneira, foi improvável buscar informações ocupacionais das gestantes adolescentes durante o período do pelo estudo.

As buscas foram realizadas pelos próprios pesquisadores em prontuários físicos e eletrônicos de todas as 15 UBSs do município, após consentimento da Secretaria Municipal de Saúde e aprovação do Comitê de Ética e Pesquisa (CEP) sob o parecer número 4.444.659 da Universidade de Gurupi (UNIRG). Os artigos selecionados para discussão e citações obedeceram a um marco temporal recente. Os resultados estão apresentados sob forma de tabelas e discussões. 


\section{RESULTADOS E DISCUSSÃO}

Os resultados apresentados nas tabelas abaixo, estão descritos em números absolutos e relativos. Referem-se aos dados encontrados nos prontuários das gestantes adolescentes atendidas durante a pandemia, exclusivamente, nas UBS da zona urbana do município em questão.

Vale salientar que na apresentação de dados percentuais, o "Não Consta", fidedignos nas variáveis encontradas nos prontuários físicos e eletrônicos através do instrumento de pesquisa, contrapõe a importância da coleta de informações básicas para uma boa abertura e acompanhamento do pré-natal, dificultando uma possível resolução de problemas que podem acarretar no futuro desse público-alvo, como por exemplo, o planejamento familiar, a desestrutura financeira, a evasão escolar e a pobreza.

Tabela 1 - Incidência de Gravidez na Adolescência nas Unidades Básicas de Saúde, n= 771.

\begin{tabular}{ccccc}
\hline UBS & \multicolumn{2}{c}{ Total de Gestantes } & \multicolumn{2}{c}{ Total de Gestantes Adolescentes } \\
\hline- & $\mathbf{N}$ & $\%$ & $\mathbf{N}$ & $\%$ \\
\hline I & 136 & $17,88 \%$ & 29 & $21,98 \%$ \\
II & 22 & $2,89 \%$ & 0 & $0 \%$ \\
III & 54 & $7,10 \%$ & 9 & $6,82 \%$ \\
IV & 56 & $7,36 \%$ & 10 & $7,58 \%$ \\
V & 27 & $3,54 \%$ & 3 & $2,28 \%$ \\
VI & 54 & $7,10 \%$ & 5 & $3,79 \%$ \\
VII & 18 & $2,36 \%$ & 1 & $0,76 \%$ \\
VIII & 42 & $5,52 \%$ & 5 & $3,73 \%$ \\
IX & 95 & $12,49 \%$ & 22 & $16,67 \%$ \\
X & 35 & $4,59 \%$ & 4 & $3,03 \%$ \\
XI & 62 & $8,14 \%$ & 13 & $9,85 \%$ \\
XII & 73 & $9,59 \%$ & 16 & $12,13 \%$ \\
XIII & 40 & $5,25 \%$ & 8 & $6,07 \%$ \\
XIV & 17 & $2,24 \%$ & 3 & $2,28 \%$ \\
XV & 30 & $3,95 \%$ & 4 & $3,03 \%$ \\
Total & 761 & $100 \%$ & 132 & $17,34 \%$ \\
\hline
\end{tabular}

Fonte: Sá DR, et al., 2021.

Nos dias de hoje a GA é vista como um problema social e de saúde pública. Este estudo revela os dados da incidência de grávidas adolescentes do município, em ano de pandemia, sendo uma fonte de dados importante para o trabalho da enfermagem e demais profissionais da saúde.

Quanto à incidência de GA, os resultados mostraram um total de 761 gestantes que fizeram acompanhamento pré-natal nas UBSs desse município, sendo que, $17,34 \%$ foram de gestantes adolescentes (Tabela 1).

Segundo o Sistema de Informações sobre Nascidos Vivos (SINASC) do MS, no ano de 2019, o município apresentou total de 1.331 gestantes, das quais $14,27 \%$ eram adolescentes. Paiva AM, et al. (2020) descreveram em seu estudo sobre os fatores de risco para a gravidez em adolescentes, na faixa etária de 10 a 19 anos, na Unidade de Referência Especializada Materno Infantil que, em 2011, revelou o número de nascimentos por ocorrência e idade da mãe de acordo com as regiões brasileiras, o Norte ficou em segundo lugar em relação à ocorrência de gravidez na adolescência, de acordo com os dados levantados, de um total de $73,49 \%$ partos de mulheres com 20 anos ou mais, e 26,49\% tinham entre 10 e 19 anos. No Tocantins, no ano de 2019 houve uma taxa de $81,20 \%$ de partos de mães não adolescentes, sendo $18,80 \%$ de partos em que as mães tinham idade entre 10 e 19 anos. Enquanto que no ano de 2020, 81,62\% do total de partos de mães não adolescentes, sendo 18,38\% nascimentos de mães com idade entre 10 e 19 anos (BRASIL, 2020). 
Tabela 2 - Características Biológicas das Adolescentes Gestantes nas Unidades Básicas de Saúde.

\begin{tabular}{|c|c|c|c|c|c|c|c|c|c|c|c|c|c|c|c|}
\hline \multirow{2}{*}{ Variáveis } & \multicolumn{15}{|c|}{ Respostas } \\
\hline & $I$ & II & III & IV & $\mathbf{V}$ & VI & VII & VIII & IX & $\mathbf{X}$ & $\mathbf{X I}$ & XII & XIII & XIV & XV \\
\hline Idade & \multicolumn{15}{|c|}{$\%$} \\
\hline $\begin{array}{l}10 \text { a } 12 \\
\text { anos }\end{array}$ & 0 & 0 & 0 & 0 & 0 & 0 & 0 & 0 & 4,55 & 0 & 0 & 0 & 0 & 0 & 0 \\
\hline $\begin{array}{l}13 \text { a } 15 \\
\text { anos }\end{array}$ & 13,8 & 0 & 0 & 40 & 0 & 0 & 0 & 0 & 9,09 & 0 & 15,3 & 6,25 & 0 & 33,3 & 75 \\
\hline $\begin{array}{l}16 \text { a } 18 \\
\text { anos }\end{array}$ & 60,1 & 0 & 44,4 & 50 & 0 & 60 & 100 & 40 & 54,5 & 75 & 68,2 & 87,5 & 87,5 & 33,3 & 0 \\
\hline 19 anos & 24,1 & 0 & 55,5 & 10 & 100 & 40 & 0 & 60 & 31,8 & 25 & 15,4 & 6,2 & 12,5 & 33,3 & 25 \\
\hline Raça & \multicolumn{15}{|c|}{$\%$} \\
\hline Branca & 0 & 0 & 22,2 & 20 & 66,7 & 0 & 0 & 20 & 9,1 & 0 & 7,7 & 6,2 & 12,5 & 0 & 0 \\
\hline Preta & 6,9 & 0 & 11,1 & 0 & 0 & 0 & 100 & 0 & 18,1 & 0 & 7,7 & 0 & 12,5 & 33,3 & 25 \\
\hline Parda & 10,3 & 0 & 44,4 & 20 & 0 & 40 & 0 & 80 & 9,1 & 25 & 69,2 & 56,2 & 50 & 0 & 0 \\
\hline Amarela & 0 & 0 & 0 & 0 & 33,3 & 0 & 0 & 0 & 0 & 0 & 15,4 & 0 & 0 & 0 & 0 \\
\hline Não Consta & 82,7 & 0 & 22,2 & 60 & 0 & 60 & 0 & 0 & 63,6 & 75 & 0 & 31,2 & 25 & 66,7 & 75 \\
\hline
\end{tabular}

Fonte: Sá DR, et al., 2021.

Em relação a faixa etária, 59,85\%, das adolescentes encontravam-se entre 16 e 18 anos de idade, 26,52\% com 19 anos, $12,88 \%$ entre 13 e 15 anos e $0,75 \%$ entre 10 e 12 anos de idade. No presente estudo, observouse que a maior prevalência de gestações na adolescência ocorreu entre 16 e 18 anos, idades consideradas baixas, uma vez que, essas adolescentes ainda não possuem maturidade para este acontecimento, que traz consigo inúmeras transformações (Tabela 2).

Em artigo produzido por Santos CKB e Silva JC (2018) para identificar os fatores que influenciam a GA nas produções científicas, afirmam que as transformações ocorridas durante a puberdade fazem com que os adolescentes vivenciem a sexualidade de maneira intensa, o que na grande maioria das vezes tem como consequência a prática sexual desprotegida, o que resulta em grande parte em uma gravidez não planejada e uma mãe despreparada.

No ano de 2019, o município estudado, contabilizou 97,37\% de adolescentes gestantes com idades de 15 a 19 anos, e 2,63\% de 10 a 14 anos (BRASIL, 2019). Em um estudo que objetivava discutir sobre as consequências da gravidez na adolescência na cidade de São Francisco do Conde-BA, foi evidenciado que os fatores que levavam à gestação nos anos iniciais da vida reprodutiva eram de natureza objetiva e subjetiva sendo os mais elencados: o desconhecimento dos métodos contraceptivos, a dificuldade das adolescentes em negociar o uso do preservativo, ingenuidade, desejo de estabelecer uma relação mais estável com o parceiro ,forte desejo pela maternidade com expectativas de mudanças de "status social" (CONCEIÇÃO RA e ALVES AMCV, 2018).

Quanto a raça das gestantes estudadas, em $49,24 \%$ dos casos, não constava a informação, $30,30 \%$ eram pardas, $9,10 \%$ pretas, $9,10 \%$ brancas e $2,28 \%$ amarelas. No ano de 2019 a cidade apresentou $74,74 \%$ de adolescentes pardas, $13,17 \%$ brancas, $8,42 \%$ pretas e $2,63 \%$ amarelas segundo dados do SINASC (Tabela 2).

De acordo com Almeida AHV (2018) em seu estudo para analisar o perfil das puérperas adolescentes no Brasil segundo desigualdades raciais e socioeconômicas, expôs que, no que tange à cor da pele, mesmo quando há acesso e utilização dos serviços de pré-natal, a assistência não se dá de forma equânime. Pobres, pretas e seus bebês apresentam resultados desfavoráveis do que o restante da população, o que pode refletir na presença de outras desvantagens sociais, ambientais ou nutricionais, apontando a necessidade de ampliar o cuidado daquelas que estão sob risco de receber assistência pré-natal inadequada. As gestantes que se declaram pretas realizam menos exames, quando comparadas às pardas e brancas, além de peregrinarem mais para ter acesso à assistência no momento do parto. 
Tabela 3 - Características Sociais das Adolescentes Gestantes nas Unidades Básicas de Saúde.

\begin{tabular}{|c|c|c|c|c|c|c|c|c|c|c|c|c|c|c|c|}
\hline \multirow{2}{*}{ Variáveis } & \multicolumn{15}{|c|}{ Respostas } \\
\hline & $I$ & II & III & IV & $\mathbf{V}$ & VI & VII & VIII & IX & $\mathbf{X}$ & $\mathbf{X I}$ & XII & XIII & XIV & $\mathrm{XV}$ \\
\hline Escolaridade & \multicolumn{15}{|c|}{$\%$} \\
\hline$\overline{\mathrm{EFI}^{*}}$ & 20,7 & 0 & 20,7 & 20 & 33,3 & 0 & 0 & 20 & 13,5 & 0 & 7,7 & 31,2 & 37,5 & 0 & 50 \\
\hline $\mathrm{EFC}^{\star \star}$ & 24,1 & 0 & 24,1 & 40 & 0 & 0 & 0 & 0 & 4,5 & 25 & 23,1 & 0 & 0 & 0 & 25 \\
\hline $\mathrm{EMI}^{\star \star \star}$ & 13,8 & 0 & 13,8 & 10 & 0 & 0 & 0 & 0 & 31,8 & 50 & 0 & 6,25 & 0 & 0 & 0 \\
\hline $\mathrm{EMC}^{\star \star \star \star \star}$ & 6,9 & 0 & 6,9 & 0 & 0 & 40 & 0 & 20 & 4,55 & 0 & 7,69 & 6,2 & 25 & 0 & 0 \\
\hline Analfabeta & 0 & 0 & 0 & 0 & 0 & 0 & 0 & 0 & 0 & 0 & 0 & 0 & 0 & 0 & 0 \\
\hline Não Consta & 34,5 & 0 & 34,5 & 30 & 66,7 & 60 & 100 & 60 & 45,5 & 25 & 61,6 & 56,2 & 37,5 & 100 & 25 \\
\hline Estado Civil & \multicolumn{15}{|c|}{$\%$} \\
\hline Solteira & 48,3 & 0 & 88,9 & 20 & 66,7 & 20 & 0 & 0 & 40,9 & 50 & 23,1 & 50 & 37,5 & 33,3 & 75 \\
\hline Casada & 0 & 0 & 0 & 0 & 33,3 & 0 & 0 & 40 & 4,5 & 25 & 0 & 6,2 & 25 & 0 & 0 \\
\hline União Estável & 34,5 & 0 & 11,1 & 20 & 0 & 40 & 10025 & 0 & 13,6 & 0 & 0 & 12,5 & 25 & 0 & 25 \\
\hline Namora & 0 & 0 & 0 & 0 & 0 & 0 & 0 & 0 & 4,5 & 25 & 0 & 0 & 0 & 0 & 0 \\
\hline Não Consta & 17 & 0 & 0 & 60 & 0 & 40 & 0 & 60 & 36,4 & 0 & 76,9 & 31,2 & 12,5 & 66,7 & 0 \\
\hline
\end{tabular}

Legenda: *Ensino Fundamental Incompleto; ${ }^{* *}$ Ensino Fundamental Completo; ${ }^{* * *}$ Ensino Médio Incompleto; ${ }^{* * * \star}$ Ensino Médio Completo. Fonte: Sá DR, et al., 2021.

Com relação ao grau de escolaridade, em $43,18 \%$ não consta a informação, $18,93 \%$ possuíam ensino fundamental incompleto, $13,64 \%$ ensino fundamental completo, $12,87 \%$ ensino médio incompleto, $11,37 \%$ ensino médio completo e analfabeta $0 \%$. A gravidez na adolescência tem impacto negativo no que diz respeito a escolaridade dessas jovens, continuar os estudos se torna um desafio, seja pela pressão social, medo de rejeição dos colegas, falta de apoio familiar ou aumento de suas responsabilidades agora com o filho as quais não estava preparada (Tabela 3).

Segundo Montenegro MA, et al. (2020) neste período, muitas adolescentes desistem dos estudos por pressão social e econômica, ora pela rejeição dos colegas à gravidez precoce, ora pela falta de suporte familiar no cuidado com seu filho, o que a impede do retorno aos estudos. Essa evasão escolar contribui para o inadequado grau de profissionalização, o que dificulta a possibilidade de um futuro com autonomia financeira, contribuindo para os riscos de desemprego e a perpetuação do ciclo da pobreza. Um estudo mostrou que $40 \%$ das adolescentes abandonaram a escola devido a gestação, 36\% abandonaram após o parto, $5,5 \%$ já havia concluído o ensino fundamental e não iniciaram o ensino médio e $19 \%$ concluíram o ensino médio (FERNANDE RFM, et al., 2017).

Quanto ao estado civil, o resultado foi de $41,67 \%$ solteiras, $32,58 \%$ não consta a informação, $18,18 \%$ em união estável, $6,06 \%$ casadas e $1,51 \%$ namorando. Aqui podemos ver que grande parte das adolescentes vivenciam essa fase sem o companheiro, o que é um fator relevante para que a jovem se sinta apoiada e segura, e, portanto, tenha uma gestação tranquila (Tabela 3).

Em 2019, o município em questão, apresentou um percentual de $40 \%$ de adolescentes solteiras, 50,53\% em união consensual e 8,42\% destas casadas (BRASIL, 2019). Em pesquisa realizada para comparação da evolução da gravidez em adolescentes em relação a mulheres adultas e a repercussão na vitalidade neonatal, foi descrito pelos autores que a predominância de ausência do companheiro entre as mães adolescentes influência de forma desfavorável a evolução da gravidez e aumenta os riscos de desdobramentos adversos à saúde neonatal, pois além das transformações físicas, a adolescente ainda tem de lidar com os questionamentos psicológicos normais dessa faixa etária, os quais se encontram naturalmente mais intensificados durante o ciclo gestacional (MONTENEGRO MA, et al., 2020).

Segundo Araújo RLD, et al. (2016) as principais consequências da gravidez enfrentadas pelas adolescentes, descreveram que a ausência da figura paterna pode vir a influenciar o desenvolvimento cognitivo das crianças e futuramente favorecer a ocorrência de distúrbios do comportamento nas mesmas, sendo estes considerados indicadores diretos de saúde e nutrição e indiretos da qualidade de vida da população. 
Tabela 4 - Características Obstétricas das Adolescentes Gestantes das Unidades Básicas De Saúde.

\begin{tabular}{|c|c|c|c|c|c|c|c|c|c|c|c|c|c|c|c|}
\hline \multirow{2}{*}{ Variáveis } & \multicolumn{15}{|c|}{ Respostas } \\
\hline & I & II & III & IV & $\mathbf{V}$ & VI & VII & VIII & IX & $\mathbf{X}$ & $\mathbf{X I}$ & XII & XIII & XIV & $\mathbf{X V}$ \\
\hline Gravidez & & & & & & & & $\%$ & & & & & & & \\
\hline Planejada & 17,2 & 0 & 0 & 0 & 0 & 0 & 0 & 60 & 4,5 & 50 & 0 & 6,2 & 12,5 & 0 & 50 \\
\hline $\begin{array}{l}\text { Não } \\
\text { Planejada }\end{array}$ & 44,8 & 0 & 100 & 20 & 33,3 & 40 & 0 & 40 & 68,2 & 25 & 61,5 & 6,2 & 50 & 0 & 50 \\
\hline $\begin{array}{l}\text { Não } \\
\text { Consta }\end{array}$ & 37,9 & 0 & 0 & 80 & 66,7 & 60 & 100 & 0 & 27,3 & 25 & 38,5 & 87,5 & 37,5 & 100 & 0 \\
\hline $\begin{array}{l}\text { Aborto } \\
\text { Anterior }\end{array}$ & & & & & & & & $\%$ & & & & & & & \\
\hline Sim & 6,7 & 0 & 0 & 10 & 0 & 0 & 0 & 20 & 13,6 & 0 & 0 & 0 & 0 & 0 & 0 \\
\hline Não & 48,3 & 0 & 100 & 50 & 100 & 60 & 100 & 60 & 72,7 & 100 & 61,5 & 75 & 87,5 & 33,3 & 100 \\
\hline $\begin{array}{l}\text { Não } \\
\text { Consta }\end{array}$ & 44,8 & 0 & 0 & 40 & 0 & 40 & 0 & 20 & 13,6 & 0 & 38,5 & 25 & 12,5 & 66,7 & 0 \\
\hline
\end{tabular}

Fonte: Sá DR, et al., 2021.

No que se refere ao planejamento da gravidez, 45,45\% das adolescentes não planejaram a gestação, 43,19\% não consta a informação e 11,36\% planejaram a gravidez (Tabela 4 ).

Segundo Araújo RLD, et al. (2016) o não planejamento da gravidez é responsável por uma série de agravos ligados a saúde reprodutiva materna e perinatal e apresenta risco de ansiedade e depressão, portanto a ocorrência acarreta impactos importantes na oferta dos cuidados do pré-natal, orientação sobre o aleitamento materno e nas taxas de morbimortalidade infantil.

Quanto a abortos anteriores, $70,45 \%$ não haviam sofrido nenhum aborto, 23,49\% não consta a informação e 6,06\% sim, sofreram aborto (Tabela 4).

Oliveira AMD (2016) em seu estudo com objetivo de desenvolver um plano de ação que visa evitar ou adiar a gestação precoce diz que, o aborto espontâneo, parto prematuro, diabetes gestacional, dificuldade de amamentação, restrição de crescimento intrauterino e depressão pós-parto são complicações de maior recorrência em gestantes adolescentes. Maus tratos, descuidos, desnutrição e acidentes domiciliares também ocorrem mais em crianças filhas de mães adolescentes. O medo e as incertezas levam muitas adolescentes a procurar um aborto clandestino. As curetagens decorrentes de abortos mal feitos aumentam proporcionalmente ao surgimento de casos de gravidez nessa faixa etária.

Considerando os dados acima, os índices de gravidez na adolescência são bastantes significativos. Para Zachi M, et al. (2016), além de constituir um problema social, no tocante da saúde, é válido salientar a dificuldade para realizar um pré-natal adequado, sobretudo as adolescentes mais jovens, que grande parte escodem a gestação e só procuram esse serviço tardiamente.

Carvalho CC (2013) completa relatando em seu artigo que, a gravidez na adolescência além de gerar consequências biológicas para a mãe e para o bebê, traz consequências sociais e psicológicas. São elas: limitação de oportunidades vocacional, evasão escolar, pobreza, deficiência financeira. Esses fatores implicam em todo processo na construção do planejamento familiar de alta qualidade.

O planejamento de forma integral é fator básico para o atendimento de adolescentes e jovens, tanto do ponto de vista da organização de serviços de apoio, prevenção, atendimento de agravos e doenças, reabilitação, quanto do entendimento dos aspectos biopsicossociais que permeiam as necessidades de saúde desses grupos. Portanto, a integralidade da assistência é um ponto primordial na atuação do enfermeiro frente a saúde sexual e reprodutiva dos adolescentes e jovens (SEHNEM DG, et al., 2019).

Conforme evidencias científicas, acredita-se que promover o autocuidado, apoiando a autonomia e independência do adolescente, é uma das principais tarefas da equipe de saúde e a interação desse público em grupos educacionais e terapêuticos podem promover isso. Além de ações educativas sobre planejamento 
familiar, incluindo a questão de gênero, a fim de reduzir o atual desequilíbrio de poder entre os sexos; desenvolver grupos educativos com a participação efetiva desse público; e trabalhar a questão da gravidez, o que ela pode representar para a sua vida e dos seus familiares (TORRES JDRV, et al., 2018).

\section{CONCLUSÃO}

Observou-se um número relevante de casos de GA durante a pandemia, pois imaginava-se que as medidas de restrição, distanciamento e o estado de quarentena, seriam medidas dificultadoras no processo de gravidez entre adolescentes, porém os números evidenciaram que tais fatores não foram determinantes neste processo. Mais pesquisas sobre este assunto fazem-se necessárias a fim de avaliar de forma concisa a problemática que se configura como um problema de saúde pública. Além disso, medidas e estratégias intervencionistas devem ser desenvolvidas de forma mais contundente, com o intuito de minimizar os efeitos devastadores na vida dos adolescentes, pois são muitas as consequências vivenciadas durante e após o período gestacional. Por fim, entende-se a necessidade da aplicação das políticas públicas envolvendo gestores, equipes multiprofissionais de saúde e a comunidade, em prol desta temática, gerando melhores estratégias que visem a promoção e a prevenção de casos de GA e exaltem a necessidade de seguimento do planejamento familiar de forma eficaz e saudável.

\section{REFERÊNCIAS}

1. ALMEIDA AHV. Gravidez e parto em adolescentes no Brasil: desigualdades raciais e socioeconômicas na assistência pré-natal e associação com nascimento prematuro. Tese (Doutorado em Epidemiologia em Saúde Pública) - Escola Nacional de Saúde Pública Sergio Arouca, Fundação Oswaldo Cruz, Rio de Janeiro, 2018; 100 p.

2. ARAÚJO RLD, et al. Gravidez na adolescência: consequências centralizadas para a mulher. Temas em Saúde, 2016 ; 16(2): 567-587.

3. BRASIL. Decreto no 10.211, de 30 de Janeiro de 2020. 2020. Disponível em: https://pesquisa.in.gov.br/imprensa/jsp/visualiza/index.jsp?data=30/01/2020\&jornal=600\&pagina=1.Acessado em: 12 de maio de 2021.

4. BRASIL. Integra Saúde Tocantins. Pré Natal, Parto e Nascimento relacionados à Gravidez na Adolescência. 2021. Disponível em: http://integra.saude.to.gov.br/Paineis/GravidezCompare. Acessado em: 11 de maio de 2021.

5. BRASIL. Sistema de Informações sobre Nascidos Vivos (SINASC). Disponível em: http://tabnet.datasus.gov.br/cgi/deftohtm.exe?sinasc/cnv/nvto.def. Acessado em: 11 de maio de 2021.

6. BRASÍLIA. Lei no 8.069, de 13 de julho de 1990. Dispõe sobre o Estatuto da Criança e do Adolescente e dá outras providências. Diário Oficial da União: seção 1, Brasília - DF, 1990. Acesso em: 03 de fevereiro de 2022.

7. CARVALHO CC. Gravidez na Adolescência: principais causas e consequências. Trabalho de Conclusão de Curso (Especialização em Atenção Básica e Saúde da Família) - Universidade Federal de Minas Gerais, Governador Valadares, 2013; $27 \mathrm{p}$.

8. CONCEIÇÃO RA, ALVES AMCV. Gravidez na Adolescência. Trabalho de Conclusão de Curso (Especialização em Saúde da Família) - Instituto de Educação a Distância, Universidade da Integração Internacional da Lusofonia AfroBrasileira, São Francisco do Conde, 2018; 17 p.

9. COSTA MIF, et al. Determinantes sociais de saúde e vulnerabilidades às infecções sexualmente transmissíveis em adolescentes. Revista Brasileira de Enfermagem, 2019; 72(6): 1595-1601.

10. CUNHA ACS, et al. Efeitos psicossociais da gravidez na adolescência: um estudo transversal. Brazilian Journal of Development, 2020; 6(7): 47412-47424.

11. FERNANDES RFM, et al. MATERNIDADE NA ADOLESCÊNCIA: MOTIVOS PARA PLANEJÁ-LA. Rev enferm UFPE on line. Recife, 11(5):1776-82, maio., 2017.

12. FLORÊNCIO JÚNIOR PG, et al. Isolamento social: consequências físicas e mentais da inatividade física em crianças e adolescentes. Revista Brasileira de Atividade Física \& Saúde, 2020; 25(1): 1-2.

13. GROSSKLANS VK. Gravidez na Adolescência: Reduzir o número de adolescentes grávidas e melhorar o acompanhamento no pré-natal com profissionais qualificados. Trabalho de Conclusão de Curso (Especialização em Saúde da Família) - Universidade do Pará, Belém, 2019; 31 p.

14. MINISTÉRIO DA SAÚDE. Secretaria de Atenção à Saúde. Área de Saúde do Adolescente e do Jovem. Marco legal: saúde, um direito de adolescentes. Editora do Ministério da Saúde. Brasília - DF: Brasil, 2007. 60 p. Disponível em: <http://bvsms.saude.gov.br > bvs > publicacoes>. Acesso em: 03 de fevereiro de 2022.

15. MONTENEGRO MA, et al. Estudo comparativo de gravidez em adolescentes e em adultas no Brasil: 2013 a 2017. Brazilian Journal of Development, 2020; 6(8): 58102-58110. 
16. OLIVEIRA AMD. Gravidez na adolescência e seus fatores críticos na área de abrangência do PSF São Sebastião. Trabalho de Conclusão de Curso (Especialização em Atenção Básica em Saúde da Família) - Universidade Federal Do Triângulo Mineiro, Conselheiro Lafaiete, Minas Gerais, 2016; 23 p.

17. OLIVEIRA WA, et al. A saúde do adolescente em tempos da COVID-19: scoping review. Cadernos de Saúde Pública, 2020; 36(8): 1-14.

18. ORGANIZAÇÃO MUNDIAL DA SAÚDE. A gravidez na adolescência. 2021. Disponível em: http://www.who.int/mediacentre/factsheets/fs364/en/. Acessado em: 12 de janeiro de 2022.

19. PAIVA AM, et al. Fatores que propiciam a gravidez na adolescência em uma unidade de referência especializada materno infantil na região Norte do Brasil: um estudo piloto. Revista Eletrônica Acervo Saúde, 2020; 49(supl. 49): e3342.

20. SANTOS CKB, SILVA JC. Fatores que influenciam a gravidez na adolescência. Trabalho de Conclusão de Curso (Graduação em Enfermagem) - Centro Universitário São Lucas, Porto Velho, 2018; 22 p.

21. SEHNEM DG, et al. Saúde sexual e reprodutiva dos adolescentes: percepções dos profissionais em enfermagem. Av Enferm. 2019; 37(3): 343-352. Disponível em: http://doi.org/10.15446/av.enferm.v37n3.78933

22. SILVA PM, MILANI DRC. Gravidez na adolescência: um problema social. Pedagogia em Foco, 2019; 14(11): 111126.

23. TORRES JDRV, et al. O significado da maternidade para adolescentes atendidas na Estratégia de Saúde da Família. Rev Fun Care Online. 2018 out/dez; 10(4):1003-1013.

24. WAGNER A. Vulnerabilidades para gestantes e puérperas durante a pandemia da COVID-19 no estado de Santa Catarina, Brasil. Hygeia-Revista Brasileira de Geografia Médica e da Saúde, 2020; 1(1): 398-406.

25. ZACHI M, et al. Maternidade na adolescência: ressignificando a vida? J Hum Growth Dev. 2016; 26(2): $199-204$. 